GEOLOGICAL SURVEY CIRCULAR 74

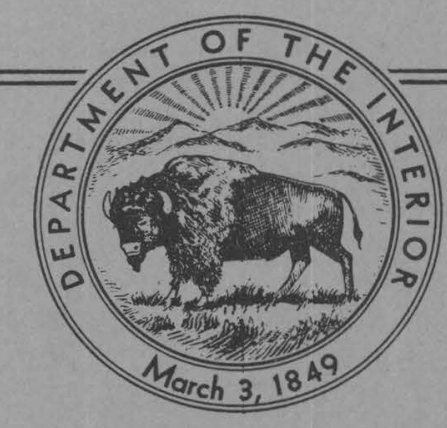

April 1950

\title{
A GLOSSARY OF URANIUM-
}

\section{AND THORIUM-BEARING MINERALS}

\author{
By \\ Judith Weiss Frondel \\ and \\ Michael Fleischer
}




\section{UNITED STATES DEPARTMENT OF THE INTERIOR Oscar L. Chapman, Secretary GEOLOGICAL SURVEY \\ W. E. Wrather, Director}

WASHINGTON, D. C. 


\title{
A GLOSSARY OF URANIUM- AND THORIUM-BEARING MINERALS
}

By

\author{
Judith Weiss Frondel \\ and \\ Michael Fleischer
}

\section{CONTENTS}

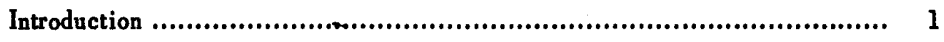

A. Uranium and thorium minerals ........................................... 2

B. Minerals with minor amounts of uranium and thorium ........................ 10

C. Minerals that should be tested for uranium and thorium ...................... 14

D. Minerals that are non-uranium- or non-thoriumbearing, but that have been reported to contain impurities or intergrowths of uranium, thorium, or rare-earth minerals ............................................ 16

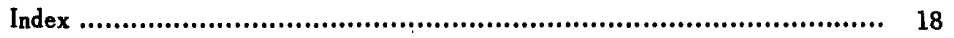


- 


\section{INTRODUCTION}

The U. S. Geological Survey has for some time been making a systematic survey of data pertaining to uranium and thorium minerals and to those minerals that contain traces or more of uranium and thorium. This survey consists of collecting authoritative chemical, optical, and $\mathrm{X}$-ray diffraction data from the literature and of adding to these data, where inadequate, by work in the laboratory. The results will be reported from time to time, and the authors welcome information on additional data and names.

The first of a series of reports on these results is this glossary of uranium- and thoriumbearing minerals. This project is part of the work on radioactive materials being done by the Geological Survey on behalf of the Atomic Energy Commission. The glossary is divided into four groups: A, minerals containing uranium and thorium as major constituents; $B$, minerals containing minor amounts of uranium and thorium; $\mathrm{C}$, minerals that, if investigated by modern analytical methods, might show urahium or thorium content; and $D$, minerals that are nonuranium- or non-thorium-bearing, but that have. been reported to contain impurities or intergrowths of uranium, thorium, or rare-earth minerals. Uranium is more widespread in its natural occurrence than generally has been supposed, and it is possible that the third group of minerals will give valuable information upon reinvestigation.

Such a glossary has long been needed to alleviate the confusion that obscures the nomenclature of these minerals. For many of them the confusion will remain until more thorough research is done. Some species always may be subject to question as their type specimens have been lost and existing specimens bearing the species name have been found to be other substances (e.g., in group A, uranochalcite and voglianite). Structural formulas are given for most of the minerals. Oxide formulas are given where no good structural data are available. The glossary summarizes the state of our knowledge, as of March 1950, concerning the validity of all the included species. Identities and group relations are indicated. It might be well to relegate to obscurity the many synonyms existing in the literature.

In the index there are 325 entries that represent 81 species containing uranium and thorium as major constituents, 39 species containing small amounts of uranium and thorium, 29 species that should be tested for uranium and thorium, and 13 species that have been reported to contain impurities or intergrowths of uranium, thorium, or rare-earth minerals. These four categories are shown in the index by reference letters.

For most of the minerals a reference has been chosen from standard reference books and easily available journals. Dana VI and Dana VII stand for the 6th and 7th editions, respectively, of Dana's System of Mineralogy.

The authors wish to thank Dr. Clifford Frondel of the Department of Mineralogy and Petrography, Harvard University, and Professor Esper S. Larsen, Jr., of the U. S. Geological Survey, for their suggestions and critical reading of the manuscript. 


\section{A. URANIUM AND THORIUM MINERALS}

\section{AMPANGABEITE}

$(\mathrm{Y}, \mathrm{Er}, \mathrm{U}, \mathrm{Ca}, \mathrm{Th})_{2}(\mathrm{Cb}, \mathrm{Ta}, \mathrm{Fe}, \mathrm{Ti})_{7} \mathrm{O}_{1}$ ?

Validity of species is questionable; may be an inhomogeneous alteration product.

$\mathrm{U}=17.1 \%, \mathrm{Th}=1.8 \%$.

Dana VII, pp. 806-807

Hydroe uxenite, a synonym of ampangabeite.

Dana VII, p. 806.

\section{ANDERSONITE}

$\mathrm{Na}_{2} \mathrm{Ca}\left(\mathrm{UO}_{2}\right)\left(\mathrm{CO}_{3}\right)_{3} \cdot 6 \mathrm{H}_{2} \mathrm{O}$

$\mathrm{U}=39.15 \%$

Milton, C., U. S. Geol. Survey, personal communication.

\section{AUTUNITE}

$\mathrm{Ca}\left(\mathrm{UO}_{2}\right)_{2}\left(\mathrm{PO}_{4}\right)_{2} \cdot 8-12 \mathrm{H}_{2} \mathrm{O}$

$\mathrm{U}=49.7$ to $56.2 \%$

Dana VI, pp. 857-858

Calcouranite, a synonym of autunite.

Meta-autunite is the hydrate with $\mathbf{8 H}_{2} \mathrm{O}$.

\section{BASSETITE}

$\mathrm{Fe}\left(\mathrm{UO}_{2}\right)_{2}\left(\mathrm{PO}_{4}\right)_{2} \cdot 8 \mathrm{H}_{2} \mathrm{O}$

$\mathrm{U}=51 \%$

Mineralog. Mag., vol. 17, pp. 221-236, 1915

Chemie der Erde, vol. 12, pp. 433-450, $1939-40$.

Frondel, C., Harvard University, personal communication.

\section{BAYLEYITE}

$\mathrm{Mg}_{2}\left(\mathrm{UO}_{2}\right)\left(\mathrm{CO}_{3}\right)_{3} \cdot 18 \mathrm{H}_{2} \mathrm{O}$

$\mathrm{U}=27.8$ to $29.2 \%$.

Milton, C., U. S. Geol. Survey, personal communication.

\section{BECQUERELITE}

$2 \mathrm{UO}_{3} .3 \mathrm{H}_{2} \mathrm{O}$ ?

$\mathrm{U}=\mathbf{8 2 . 4 \%}$

Dana VII, pp. 625-627

Billietite, a variety of becquerelite.

Am, Mineralogist, vol. 33, pp. 503-507, 1948.

\section{BETAFITE}

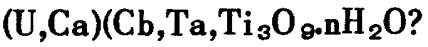

$\mathrm{U}=16.3$ to $24.5 \%$.

$\mathrm{Th}=1.0$ to $1.1 \%$

Dana VII, pp. 803-805

Blomstrandite, a synonym of betafite.

$U=16.3 \%$

Dana VII, pp. 803-805.

Mendeleyevife, titanian betafite.

$\mathrm{U}=13.7 \%$

Dana VII, pp. 803-804.

Samiresite, a synonym of betafite.

$\mathrm{U}=18.7 \%$

Dana VII, pp. 803-805.

\section{BRANNERITE}

(U,Ca,Fe, Y,Th) ${ }_{3} \mathrm{Ti}_{5} \mathrm{O}_{18}$ ?

$\mathrm{U}=39.3 \%, \mathrm{Th}=3.6 \%$

Dana VII, pp. 774-775.

CALCIOSAMARSKITE

(Ca,Y,etc.,U,Th) ${ }_{3}(\mathrm{Cb}, \mathrm{Ta}, \mathrm{Fe}, \mathrm{Ti}, \mathrm{Sn})_{5} \mathrm{O}_{15}$

$(\mathrm{Ca}, \mathrm{Y} \text {,etc.,U,Th, Zr })_{3}(\mathrm{Cb}, \mathrm{Ta}, \mathrm{Fe}, \mathrm{Ti})_{5} \mathrm{O}_{16}$

$\mathrm{U}=9.4$ to $11.3 \%$

$\mathrm{Th}=1.9$ to $2.9 \%$

Dana VII, p. 772

\section{CARNOTITE}

$\mathrm{K}\left(\mathrm{UO}_{2}\right)\left(\mathrm{VO}_{4}\right) \cdot 1 \frac{1}{2}-1 \frac{1}{2} \mathrm{H}_{2} \mathrm{O}$

$\mathrm{U}=\mathbf{5 6 . 3}$ to $58.4 \%$

Dana VI, Appendix I, pp. 13-14

\section{CLARKEITE}

$\mathrm{UO}_{3} \cdot \mathrm{nH}_{2} \mathrm{O}$ ?

$\mathrm{U}=\mathbf{7 3 . 7 \%}$

Dana VII, pp. 624-625

\section{CUPROSKLODOWSKITE}

$\mathrm{Cu}\left(\mathrm{UO}_{2}\right)_{2} \mathrm{Si}_{2} \mathrm{O}_{7} .6 \mathrm{H}_{2} \mathrm{O}$

$\mathrm{U}=58.6 \%$

Am. Mineralogist, vol. 19, p. 235, 1934 
Jachymovite = cuprosklodowskite

Mineralog. Abs., vol. 6, p. 345, 1936

Uranochalcite - a hydrous copper, uranium, and calcium sulfate of questionable validity.

Voglianite - A hydrous calcium and uranium sulfate of questionable validity. The type material of Vogl for uranochalcite and voglianite has not been found. Present museum specimens bearing these names have proved to be cuprosklodowskite:

Vogl, J. F., Gangverhältnisse and Mineralreichtum Joachimthals, Teplitz, 1857 Ceské Spol. Nauk Vestnik, article VII, pp. 1-36, 1935

\section{CURITE}

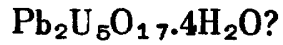

$\mathrm{U}=66.2$ to $70.1 \%$

Dana VII, pp. 629-631

\section{DELORENZITE}

$\left(\mathrm{Y}, \mathrm{U}, \mathrm{Fe}^{2}\right)(\mathrm{Ti}, \mathrm{Sn})_{3} \mathrm{O}_{8}$ ?

$\mathrm{U}=\mathbf{8 . 7 \%}$

Dana VII, p. 808

\section{DEWINDTITE}

$\mathrm{Pb}\left(\mathrm{UO}_{2}\right)_{2}\left(\mathrm{PO}_{4}\right)_{2} .3 \mathrm{H}_{2} \mathrm{O}$ ?

$\mathrm{U}=52.1 \%$

Am.,Mineralogist, vol. 7, p. 162, 1922

Stasite = dewindite? -

$\mathrm{Pb}_{4} \mathrm{U}_{8} \mathrm{P}_{6} \mathrm{O}_{43} \cdot \mathrm{nH}_{2} \mathrm{O}$ ?

Am. Mineralogist, vol. 7, pp. 196-197, 1922

\section{DIDERICHITE}

Contains uranium, water, and carbonate. Validity of species is questionable.

Soc. belge geologie Bull., vol. 70, pp. 212-225, 1947

\section{DJALMAITE}

$$
(\mathrm{U}, \mathrm{Ca}, \mathrm{Pb})\left(\mathrm{Ta}, \mathrm{Cb}, \mathrm{Ti}_{3} \mathrm{O}_{2} \cdot \mathrm{nH}_{2} \mathrm{O}\right.
$$

May be tantalum equivalent of betafite. May be microlite; Tavora, E., University of Brazil, personal communication, Oct. 1949

$\mathrm{U}=10.4 \%$

Dana VII, p. 805

\section{DROOGMANSITE}

No chemical analysis has been made. May be related to sklodowskite.

Am. Mineralogist, vol. 11, p. 168, 1926

\section{DUMONTITE}

$\mathrm{Pb}_{2}\left(\mathrm{UO}_{2}\right)_{3}\left(\mathrm{PO}_{4}\right)_{2} \mathrm{O}_{2} .5 \mathrm{H}_{2} \mathrm{O}$

$\mathrm{U}=51.0 \%$

Am. Mineralogist, vol. 10, p. 131, 1925

\section{EPIIANTHINITE}

$\mathrm{yUO}_{3} \cdot \mathrm{xH}_{2} \mathrm{O}$ ?

No good chemical analysis has been made.

An alteration product of ianthinite.

Am. Mineralogist, vol. 32, pp. 344-350, 1947

\section{ESCHY.NITE}

$\left(\mathrm{Ce}, \mathrm{Ca}, \mathrm{Fe}\right.$ ?,Th)(Ti,Cb) ${ }_{2} \mathrm{O}_{6}$

Also aeschynite. In eschynite-priorite series.

$\mathrm{Th}=9.9$ to $15.4 \%$

Dana VII, pp. 793-796

\section{EUXENITE}

$(\mathrm{Y}, \mathrm{Ca}, \mathrm{Ce}, \mathrm{U}, \mathrm{Th})(\mathrm{Cb}, \mathrm{Ta}, \mathrm{Ti})_{2} \mathrm{O}_{\odot}$

In euxenite-polycrase series.

$\mathrm{U}=\mathbf{3 . 0}$ to $\mathbf{9 . 0 \%}$

$\mathrm{Th}=$ up to $4.3 \%$

Dana VII, pp. 787-791

Lyndochite - variety of euxenite

$\mathrm{U}=0.6 \%, \mathrm{Th}=4.3 \%$

Dana VII, pp. 787, 789-791

Tanfeuxenife - variety of euxenite

$\mathrm{U}=3.0$ to $3.8 \%, \mathrm{Th}=\mathrm{tr}$.

Dana VII, pp. 787, 789-790

Eschwegife - $\mathrm{AB}_{2} \mathrm{O}_{\text {}}$

$A=Y, E r, U, T h$

$\mathrm{B}=\mathrm{Cb}, \mathrm{Ta}, \mathrm{Ti}, \mathrm{Fe}$

$\mathrm{U}=1.7 \%, \mathrm{Th}=0.5 \%$

May belong to euxenite-polycrase series.

Dana VII, pp. 792-793

Toddite - possibly a mixture of columbite and euxenite.

$\mathrm{U}=9.8 \%, \mathrm{Th}=0.4 \%$

Dana VII, pp. 785-786 


\section{FERGUSONITE}

$(\mathrm{Y}, \mathrm{Er}, \mathrm{Ce}, \mathrm{Fe})(\mathrm{Cb}, \mathrm{Ta}, \mathrm{Ti}) \mathrm{O}_{4}$

In fergusonite-formanite series.

$\mathrm{U}=0.8$ to $6.3 \%$

$\mathrm{Th}=0.7$ to $2.5 \%$

Dana VII, pp. 757-762

Adelpholite - synonym of fergusonite? A poorly defined substance, possibly an altered mossite.

Dana VII, pp. 762, 778-779

Arrhenite - an altered fergusonite

Dana VII, p. 762

Bragite - synonym of fergusonite

$\mathrm{U}=7.2 \%$

Dana VII, pp. 757, 759, 761

Kochelite - synonym of fergusonite

Dana VII, pp. 757, 761

Risörite - synonym of fergusonite

$\mathrm{U}=0.09 \%, \mathrm{Th}=\mathrm{tr}$.

Dana VII, pp. 757-758, 760-762

Rutherfordite - an altered fergusonite

Dana VII, pp. 757, 761-762

Sipylite - synonym of fergusonite

$\mathrm{U}=0.8$ to $6.3 \%$

Dana VII; pp. 757, 759-760, 762

Tyrite - synonym of fergusonite

Dana VII, pp. 757, 760-761

\section{FORMANITE}

$(\mathrm{U}, \mathrm{Th}, \mathrm{Ca})(\mathrm{Ta}, \mathrm{Cb}, \mathrm{Ti}) \mathrm{O}_{4}$

In fergusonite-formanite series.

$\mathrm{U}=1.1 \%, \mathrm{Th}=1.1 \%$

Dana VII, pp. 758, 760, 762

\section{FOURMARIERITE}

$\mathrm{PbU}_{4} \mathrm{O}_{13} .5 \mathrm{H}_{2} \mathrm{O}$ ?

$\mathrm{U}=70.1$ to $70.8 \%$

Dana VII, pp. 628-629

\section{FRIT ZSCHEITE}

$\mathrm{Mn}\left(\mathrm{UO}_{2}\right)_{2}\left[(\mathrm{P}, \mathrm{V}) \mathrm{O}_{4}\right]_{2} \cdot 8 \mathrm{H}_{2} \mathrm{O}$ ?
No actual analysis is available. May be the manganese analogue of torbernite.

Dana VI, p. 860

\section{GUMMITE}

$\mathrm{UO}_{3} \cdot \mathrm{nH}_{2} \mathrm{O}$ ?

Generic term for minerals occurring as alteration products of uraninite and not otherwise identified. Group includes silicates, phosphates, and oxides.

$\mathrm{U}=31.3$ to $70.1 \%$

$\mathrm{Th}=$ up to $22.0 \%$

Dana VII, pp. 622-625

Eliasife - synonym of gurmite

$\mathrm{U}=57.2 \%$

Dana VII, pp. 622-624

Pittinite - synonym of gummite

Dana VII, pp. 622-624

Ytrogummife - synonym of gummite

Alteration product of yttrian uraninite.

Dana VII, pp. 622-624

\section{HUTTONITE}

$\mathrm{ThSiO}_{4}$

Monoclinic dimorph of thorite.

$\mathrm{Th}=67.4 \%$

$\mathrm{U}=$ none

Pabst, Adolf, University of California, personal communication; in press Am. Mineralogist.

\section{IANTHINITE}

$2 \mathrm{UO}_{2} .7 \mathrm{H}_{2} \mathrm{O}$ ?

$\mathrm{U}=70.3 \%$

Dana VII, pp. 633-634

\section{ISHIKAWAITE}

(U,Fe,Y,etc.)(Cb,Ta) $0_{4}$

$\mathrm{U}=19.3 \%$

Dana VII, p. 766

JOHANNITE

$\mathrm{Cu}\left(\mathrm{UO}_{2}\right)_{2}\left(\mathrm{SO}_{4}\right)_{2}(\mathrm{OH})_{2} \cdot 6 \mathrm{H}_{2} \mathrm{O}$

$\mathrm{U}=55.3$ to $61.1 \%$

Am. Mineralogist, vol. 11, pp. 1-5, 1926 
Gilpinite = johannite

Am. Mineralogist, vol. 11, pp. 1-5, 1926

\section{KASOLITE}

$\mathrm{Pb}\left(\mathrm{UO}_{2}\right) \mathrm{SiO}_{4} \cdot \mathrm{H}_{2} \mathrm{O}$

$\mathrm{U}=43.6$ to $44.5 \%$

Am. Mineralogist, vol. 7, pp. 128-129, 1922

\section{KHLOPINITE}

$\left(\mathrm{Y}, \mathrm{U}^{4}, \mathrm{Th}\right)_{3}(\mathrm{Cb}, \mathrm{Ta}, \mathrm{Ti}, \mathrm{Fe})_{7} \mathrm{O}_{2}{ }^{\circ}$ ?

Also chlopinite, hlopinite

May be' related to euxenitepolycrase series.

$\mathrm{U}=7.2 \%, \mathrm{Th}=1.9 \%$

Dana VII, p. 792

\section{LIEBIGITE}

$\mathrm{Ca}_{2}\left(\mathrm{UO}_{2}\right)\left(\mathrm{CO}_{3}\right)_{3} \cdot 10 \mathrm{H}_{2} \mathrm{O}$ ?

$\mathrm{U}=\mathbf{3 4 . 3 \%}$

Dana VI, p. 308

Uranothallite - synonym of liebigite

Frondel, C., Harvard University, personal communication.

Flutherite - synonym of uranothallite

Dana VI, p. 307

\section{MACKINTOSHITE}

$(\mathrm{Th}, \mathrm{U}) \mathrm{SiO}_{4} \cdot \mathrm{H}_{2} \mathrm{O}$ ?

$\mathrm{U}=19.7 \%, \mathrm{Th}=$ up to $39.9 \%$

Dana VI, appendix I, p. 44

\section{Hydrothorite}

$\mathrm{ThSiO}_{4} .4 \mathrm{H}_{2} \mathrm{O}$

Alteration product of mackintoshite.

$\mathrm{U}=1.9 \%, \mathrm{Th}=50.7 \%$

Am. Mineralogist, vol. 13, p. 570, 1928

\section{Pilbarite}

$\mathrm{UO}_{3} \cdot \mathrm{PbO} \cdot \mathrm{ThO}_{2} \cdot 2 \mathrm{SiO}_{2} \cdot 4 \mathrm{H}_{2} \mathrm{O}$

Alteration product of mackintoshite; close to thorogummite.

$\mathrm{U}=24.4 \%, \mathrm{Th}=27.4 \%$

Am. Mineralogist, vol. 13, pp. 464-465, 1928

\section{MAITLANDITE}

$(\mathrm{U}, \mathrm{Th}, \mathrm{Pb}) \mathrm{SiO}_{4} \cdot 3 \mathrm{H}_{2} \mathrm{O}$ ?
$\mathrm{U}=31.2 \% ; \mathrm{Th}=22.7 \%$

Am. Mineralogist, vol. 16, p. 472, 1931

Nicolayite - alteration product of maitlandite; close to thorogummite.

$\mathrm{U}=33.7 \%, \mathrm{Th}=21.4 \%$

Am. Mineralogist, vol. 16, p. 409, 1931

\section{MASUYITE}

Hydrated lead uranium oxide.

Validity of species is questionable.

Soc. belge géologie Bull., vol. 70, pp. 212-225, 1947

\section{MEDJIDITE}

Sulfate of uranium and calcium.

Validity of species is questionable.

Dana VI, p. 978

\section{MONAZITE}

Essentially (Ce,La,Nd,Pr)PO 4 containing $\mathrm{ThO}_{2}$ and $\mathrm{SiO}_{2}$

$\mathrm{Th}=8 \%$ commonly; but up to $26 \%$ is reported, Davidson, C. F., Geol. Survey Great Britain, personal communication.

Dana VI, pp. 749-752

Cryptolite - synonym of monazite

Dana VI, Pp. 749, 752

Edwardsite - synonym of monazite

Dana VI, p. 749

Eremite - synonym of monazite

Dana VI, pp. 749, 752

Korarfveite - impure monazite

Dana VI, p. 752

Mengite - synonym of monazite

Dana VI, p. 749

Monazifoid - synonym of monazite

Dana VI, p. 749

Phosphocerite - synonym of monazite

Dana VI, p. 749

Tumerite - synonym of monazite

Dana VI, pp. 749, 751-752

Urdite - synonym of monazite 
NOHLITE

$(\mathrm{Ca}, \mathrm{Mg}, \mathrm{Fe}, \mathrm{Y}, \text { etc. }, \mathrm{U})_{2}\left(\mathrm{Cb}, \mathrm{Zr}, \mathrm{Fe}^{3}\right)_{3} \mathrm{O}_{10}$

Validity of species is questionable.

$\mathrm{U}=13.0 \%$

Dana VII, p. 772

\section{PARASCHOEPITE}

$\mathrm{UO}_{3} \cdot 2 \mathrm{H}_{2} \mathrm{O}$ ?

$\mathrm{U}=\mathbf{8 0 . 5 \%}$

Am. Mineralogist, vol. 32, pp. 344-350, 1947

\section{PARSONSITE}

$\mathrm{Pb}_{2}\left(\mathrm{UO}_{2}\right)\left(\mathrm{PO}_{4}\right)_{2} \cdot 2 \mathrm{H}_{2} \mathrm{O}$

$\mathrm{U}=26.8 \%$

Am. Mineralogist, vol. 8, p. 150, 1923

\section{PHOSPHURANYLITE}

A lead, calcium, uranium phosphate.

$\mathrm{U}=\mathbf{5 7 . 3 \%}$

Frondel, C., Harvard University, personal communication

\section{POLYCRASE}

$(\mathrm{Y}, \mathrm{Ca}, \mathrm{Ce}, \mathrm{U}, \mathrm{Th})(\mathrm{Ti}, \mathrm{Cb}, \mathrm{Ta})_{2} \mathrm{O}_{6}$

In euxenite-polycrase series.

$\mathrm{U}=5.5$ to $12.4 \%$

$\mathrm{Th}=$ up to $4.7 \%$

Dana VII, pp. 787-791

\section{POLYMIGNYTE}

$\mathrm{A}(\mathrm{Cb}, \mathrm{Ti}, \mathrm{Ta}) \mathrm{O}_{4}$

$\mathrm{A}=\mathrm{Ca}, \mathrm{Fe}^{2}, \mathrm{Y}, \mathrm{Zr}, \mathrm{Th}$

$\mathrm{Th}=3.4 \%$

Dana VII, pp. 764-766

\section{PRIORITE}

$\left(\mathrm{Y}, \mathrm{Er}, \mathrm{Ca}, \mathrm{Fe}^{2}, \mathrm{Th}\right)(\mathrm{Ti}, \mathrm{Cb})_{2} \mathrm{O}_{6}$

In eschynite-priorite series.

$\mathrm{U}=0.4$ to $3.4 \%$

$\mathrm{Th}=0.5$ to $14.9 \%$

Dana VII, pp. 793-796

$B$ lomstrandine - synonym of priorite Also, blomstrandinite

Dana VII, pp. 793-796

\section{RAUVITE}

$\mathrm{CaO} .2 \mathrm{UO}_{3} \cdot 6 \mathrm{~V}_{2} \mathrm{O}_{5} \cdot 2 \mathrm{OH}_{2} \mathrm{O}$

$\mathrm{U}=21.4 \%$

Am. Mineralogist, vol. 8, p. 187, 1923

U. S. Geol. Survey Bull. 750, pp. 68-70, 1935

\section{RENARDITE}

$\mathrm{Pb}\left(\mathrm{UO}_{2}\right)_{4}\left(\mathrm{PO}_{4}\right)_{2}(\mathrm{OH})_{4} \cdot 7 \mathrm{H}_{2} \mathrm{O}$

$\mathrm{U}=58.5 \%$

Am. Mineralogist, vol. 14, p. 244, 1929

\section{RICHETITE}

Contains $\mathrm{Pb}$ and $\mathrm{U}$.

Validity of species is questionable.

Soc. belge géologie Bull., vol. 70, pp. 212-225, 1947

\section{RUTHERFORDINE}

$\mathrm{UO}_{2} \mathrm{CO}_{3}$ ?

Validity of species is questionable.

$\mathrm{U}=72.1 \%$

Mineralog. Mag., vol. 14, p. 409, 1907

\section{SALÉEITE}

$\mathrm{Mg}\left(\mathrm{UO}_{2}\right)_{2}\left(\mathrm{PO}_{4}\right)_{2} \cdot 8 \mathrm{H}_{2} \mathrm{O}$

$\mathrm{U}=58.4 \%$

Am. Mineralogist, vol. 19, p. 36, 1934

SAMARSKITE

$(\mathrm{Y}, \mathrm{Er}, \mathrm{U}, \mathrm{Ca}, \mathrm{Fe}, \mathrm{Th})(\mathrm{Cb}, \mathrm{Ta})_{2} \mathrm{O}_{6}$

$\mathrm{U}=8.4$ to $16.1 \%$

$\mathrm{Th}=$ up to $3.7 \%$

Dana VII, pp. 797-800

Annerödite - mixture of samarskite and parallel growths of columbite.

Dana VII, pp. 797, 799

Eytlandite - synonym of samarskite

Dana VII, p. 797.

Hydrosamarskite - an altered samarskite

Dana VII, pp. 799-800

Plumbon iobite - a columbate of $\mathrm{Y}, \mathrm{U}, \mathrm{Pb}, \mathrm{Fe}$,etc.

May be a plumbian variety of samarskite.

Dana VII, p. 800

Rogersite - synonym of samarskite

6 Dana VII, p. 800 
Uranotantalite - synonym of samarskite

Dana VII, p. 797

Viefinghofite - synonym of samarskite

Dana VII,.pp. 800-801

Ytro-ilmenite - synonym of samarskite

Dana VII, p. 797

\section{SCHOEPITE}

$4 \mathrm{UO}_{3} \cdot 9 \mathrm{H}_{2} \mathrm{O}$ ?

$\mathrm{U}=79.0 \%$

Am. Mineralogist, vol. 8, pp. 67-69, 1923

\section{SCHROECKINGERITE}

$\mathrm{Ca}_{3} \mathrm{NaUO}_{2}\left(\mathrm{CO}_{3}\right)_{3}\left(\mathrm{SO}_{4}\right) \mathrm{F} .10 \mathrm{H}_{2} \mathrm{O}$

$\mathrm{U}=29.0 \%$

Am. Mineralogist, vol. 33, pp. 152-157, 1948

Dakeite - synonym of schroeckingerite

\section{SENGIERITE}

$\mathrm{Cu}_{2}\left(\mathrm{UO}_{2}\right)_{2}\left(\mathrm{VO}_{4}\right)_{2} \mathrm{O} \cdot 10 \mathrm{H}_{2} \mathrm{O}$ ?

$\mathrm{U}=\mathbf{4 7 . 2 \%}$

Am. Mineralogist, vol. 34, pp. 109-120, 1949

\section{SHARPITE}

$6 \mathrm{UO}_{3} \cdot 5 \mathrm{CO}_{2} \cdot 8 \mathrm{H}_{2} \mathrm{O}$ ?

Composition needs to be checked.

$\mathrm{U}=73.1 \%$

Am. Mineralogist, vol. 24, p. 658, 1939

\section{SKLODOWSKITE}

$\mathrm{Mg}\left(\mathrm{UO}_{2}\right)_{2} \mathrm{Si}_{2} \mathrm{O}_{7} .7 \mathrm{H}_{2} \mathrm{O}$

$\mathrm{U}=58.4 \%$

Am. Mineralogist, vol. 10,p. 132, 1925

Chinkolobwite - synonym of sklodowskite

Am. Mineralogist, vol. 9, p. 156, 1924

\section{SODDYITE}

$5 \mathrm{UO}_{3} \cdot 2 \mathrm{SiO}_{2} \cdot 6 \mathrm{H}_{2} \mathrm{O}$ ?

$\mathrm{U}=77.0 \%$

Mineralog. Abs., vol. 3, p. 371, 1927

\section{STUDTITE}

A hydrated carbonate of $\mathrm{U}$ and $\mathrm{Pb}$. Validity of species is questionable.

Soc. belge gèologie Bull., vol. 70, pp. 212-225 1947

\section{SWARTZITE}

$\mathrm{CaMgUO}_{2}\left(\mathrm{CO}_{3}\right)_{3} \cdot 12 \mathrm{H}_{2} \mathrm{O}$

$\mathrm{U}=33.6 \%$

Milton, C., U. S. Geol. Survey, personal communication

\section{THORIANITE}

$(\mathrm{Th}, \mathrm{U}) \mathrm{O}_{2}$

Forms series with uraninite

$\mathrm{U}=15.9$ to $39.0 \%$

$\mathrm{Th}=33.7$ to $81.5 \%$

Dana VII, pp. 620-622

Uranothorianite $=$ uranium-rich member of series

\section{THORITE}

$\mathrm{ThSiO}_{4}$

$\mathrm{U}=$ up to $9.0 \%$

$\mathrm{Th}=25.2$ to $62.7 \%$

Dana VI, pp. 488-489

Averlite - phosphatian variety of thorite

$\mathrm{Th}=60.7$ to $61.5 \%$

Dana VI, pp. $488-490$

Calciothorite - variety of thorite

$\mathrm{Th}=52.3 \%$

Dana VI, p. 489

Enalite - uranoan thorite

$\mathrm{U}=9.4 \%, \mathrm{Th}=25.4 \%$

Am. Mineralogist, vol. 18, p. 223, 1933

Eucrasife - variety of thorite

$\mathrm{Th}=31.6 \%$

Dana VI, p. 489

Ferrothorite - a ferrian thorite

$\mathrm{U}=\mathbf{2 . 4 \%}, \mathrm{Th}=\mathbf{5 4 . 0 \%}$

Am. Mineralogist, vol. 14, p. 78, 1929

Freyalite - variety of thorite

$\mathrm{Th}=25.2 \%$

Dana VI, p. 489

Hyblite - (both alpha and beta) - hydrous basic sulfo-silicate of $\mathrm{Th}$, with minor $\mathrm{U}, \mathrm{Fe}$, and $\mathrm{Pb}$. An alteration product of thorite.

Am. Mineralogist, vol. 12, pp. 368-372, 1927 
Orangite - synonym of thorite

$\mathrm{U}=1.0 \%, \mathrm{Th}=62.7 \%$

Dana VI, pp. 488-489

Uranothorife - uranoan thorite

$\mathrm{U}=7.4$ to $13.9 \%$

$\mathrm{Th}=38.2$ to $42.3 \%$

Dana VI, pp. 488-489

\section{THOROGUMMTE}

$(\mathrm{Th}, \mathrm{U}) \mathrm{SiO}_{4} \cdot 6 \mathrm{H}_{2} \mathrm{O}$ ?

Compare pilbarite and nicolayite, p. 14.

$\mathrm{U}=20.2 \%, \mathrm{Th}=36.3 \%$

Dana VI, p. 893

Chlorothorife - synonym of thorogummite

Dana VI, p. 893

\section{THOROTUNGSTITE}

$3 \mathrm{WO}_{3} \cdot \mathrm{ThO}_{2} \cdot 4 \mathrm{H}_{2} \mathrm{O}$ ?

$\mathrm{Th}=14.0 \%$

Am. Mineralogist, vol. 13, p. 159, 1928

\section{TORBERNITE}

$\mathrm{Cu}\left(\mathrm{UO}_{2}\right)_{2}\left(\mathrm{PO}_{4}\right)_{2} \cdot 8-12 \mathrm{H}_{2} \mathrm{O}$

$\mathrm{U}=\mathbf{5 1 . 2}$ to $56.0 \%$

Dana VI, pp. 856-857

Chalcolite - synonym of torbernite

Cupro-uranite - synonym of torbernite

Mefachalcolite - synonym of metatorbernite

\section{Metatorbernite}

$\mathrm{Cu}\left(\mathrm{UO}_{2}\right)_{2}\left(\mathrm{PO}_{4}\right)_{2} \cdot 8 \mathrm{H}_{2} \mathrm{O}$

$\mathrm{U}=56 \%$

Mineralog. Mag., vol. 17, pp. 326-339, 1916

Uranite $=$ torbernite-autunite group

Dana VI, pp. 856-857

Uranophyllite - synonym of torbernite

\section{TRÖGERITE}

$\left(\mathrm{UO}_{2}\right)_{3}\left(\mathrm{AsO}_{4}\right)_{2} \cdot 12 \mathrm{H}_{2} \mathrm{O}$

$\mathrm{U}=\mathbf{5 7 . 5 \%}$

Dana VI, pp. $859-860$

\section{TYUYAMUNITE}

$\mathrm{Ca}\left(\mathrm{UO}_{2}\right)_{2}\left(\mathrm{VO}_{4}\right)_{2} \cdot \mathrm{nH}_{2} \mathrm{O}$ n $=9-10$, but may be down to 4

$\mathrm{U}=47.1$ to $59.0 \%$

Am. Mineralogist, vol. 12, p. 382, 1927

Calciocarnotite - synonym of tyuyamunite

Ferghanite - probably identical with tyuyamunite, but original descriptions indicate minerals may be different.

Frondel, C., Harvard University, personal communication

\section{URACONITE}

Hydrous sulfate of $\mathrm{U}$ and $\mathrm{Cu}$.

$\mathrm{U}=61.0 \%$

Dana VI, p. 978

Uranocher - synonym of uraconite

\section{URANINITE}

$\mathrm{UO}_{2}$ (between $\mathrm{UO}_{2}$ and $\mathrm{U}_{3} \mathrm{O}_{8}$, with $\mathrm{U}^{4}$ predominant)

$\mathrm{U}=46.4$ to $97.6 \%$

$\mathrm{Th}=$ up to $12.2 \%$

Dana VII, pp. $611-620$

\section{Bröggerite}

Thorian uraninite $(\mathrm{U}, \mathrm{Th}) \mathrm{O}_{2}$

$\mathrm{U}=68.3 \%, \mathrm{Th}=5.3 \%$

Dana VII, pp. 611-614

Cleveife - uraninite with rare earths

$\mathrm{U}=57.0 \%, \mathrm{Th}=4.0 \%$

Dana VII, pp. 611, 613-614

Coracite - synonym of uraninite

Dana VII, pp. 611, 615, 617

Nastuman - synonym of pitchblende

Dana VII, pp. 611, 614, 617

Nivenite - uraninite with rare earths

$\mathrm{U}=57.8 \%^{\circ}, \mathrm{Th}=5.9 \%$

Dana VII, pp. 611, 613-615, 617

Pitchblende - colloform and massive uraninite

$\mathrm{U}=\mathbf{5 6 . 9}$ to $82.9 \%$

Dana VII, pp. $611-619$

Ulrichite - synonym of uraninite

Dana VII, pp. 611, 613, 617

Uranoniobite - synonym of uraninite

Dana VII, pp. 611, 613 
Uranopissite - synonym of uraninite

\section{URANOCIRCITE}

$\mathrm{Ba}\left(\mathrm{UO}_{2}\right)_{2}\left(\mathrm{PO}_{4}\right)_{2} \cdot 8 \mathrm{H}_{2} \mathrm{O}$

Barium analogue of autunite

$\mathrm{U}=51.3 \%$

Dana VI, p. 859

\section{URANOPHANE}

$\mathrm{Ca}\left(\mathrm{UO}_{2}\right)_{2} \mathrm{Si}_{2} \mathrm{O}_{7} \cdot 6 \mathrm{H}_{2} \mathrm{O}$

$\mathrm{U}=48.1$ to $60.4 \%$

Dana VI, p. 699

Beta-uranophane - polymorph of uranophane

Nováćek, R.,Ćeske Spol. Nauk Vèstnik, article VII, pp. 1-36, 1935

Lambertite - synonym of uranophane

Am. Mineralogist, vol. 11, pp. 155-157, 1926

Randite - a mixture of betauranophane, some tyuyamunite, and calcite.

Frondel, C., Harvard University, personal communication

Uranotil - synonym of uranophane

$\mathrm{U}=56.7$ to $60.2 \%$

Dana VI, p. 699

\section{URANOPILITE}

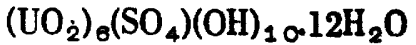

$\mathrm{U}=69.6$ to $69.9 \%$

\section{Beta-uranopilite}

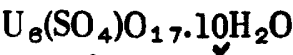

Nováćek, R., Ceské Spol. Nauk VĚatní, article VII, pp. 1-36, 1935

\section{URANOSPATHITE}

Hydrated uranyl phosphate?

Composition unknown

Mineralog. Mag., vol. 17, pp. 221-236, 1915

\section{URANOSPHAERITE}

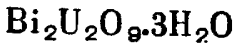

$\mathrm{U}=\mathbf{4 5 . 9}$ to $43.3 \%$

Dana VII, p. 631
URANOSPINITE

$\mathrm{Ca}\left(\mathrm{UO}_{2}\right)_{2}\left(\mathrm{AsO}_{4}\right)_{2} \cdot 8-12 \mathrm{H}_{2} \mathrm{O}$

$\mathrm{U}=53.4 \%$

Dana VI, pp. 858-859

UVANITE

$2 \mathrm{UO}_{3} \cdot 3 \mathrm{~V}_{2} \mathrm{O}_{5} \cdot 15 \mathrm{H}_{2} \mathrm{O}$

$\mathrm{U}=32.9 \%$

Mineralog. Mag., vol. 17, p. 359, 1916

Washington Acad. Sci. Jour., vol. 4, p. 576, 1914

VANDENBRANDEITE

$\mathrm{CuUO}_{4} \cdot 2 \mathrm{H}_{2} \mathrm{O}$

$\mathrm{U}=63.5$ to $64.3 \%$

Dana VII, pp. $632-633$

Uranolepidite - synonym of vandenbrandeite

Am. Mineralogist, vol. 19, pp. 235-236, 1934

VANDENDRIESSCHEITE

Hydrous lead uranium oxide Validity of species is questionable.

Soc. belge géologie Bull., vol. 70, pp. 212-225, 1947

\section{VOGLITE}

$\mathrm{Cu}_{2} \mathrm{Cu}\left(\mathrm{UO}_{2}\right)\left(\mathrm{CO}_{3}\right)_{4} \cdot 6 \mathrm{H}_{2} \mathrm{O}$ ?

$\mathrm{U}=\mathbf{3 4 . 7 \%}$

Dana VI, p. 308

\section{WALPURGITE}

$\mathrm{Bi}_{4}\left(\mathrm{UO}_{2}\right)\left(\mathrm{AsO}_{4}\right)_{2} \mathrm{O}_{4} \cdot 3 \mathrm{H}_{2} \mathrm{O}$

$\mathrm{U}=13.5$ to $17.0 \%$

Dana VI, p. 860; Neues Jahrb., 44, Abt. A, 1948; Evans, H. T.,personal communication, 1949

\section{ZEUNERITE}

$\mathrm{Cu}\left(\mathrm{UO}_{2}\right)_{2}\left(\mathrm{AsO}_{4}\right)_{2} \cdot 8 \mathrm{H}_{2} \mathrm{O}$

$\mathrm{U}=50.4 \%$

Dana VI, p. 857

\section{ZIPPEITE}

$2 \mathrm{UO}_{3} . \mathrm{SO}_{3} .5-6 \mathrm{H}_{2} \mathrm{O}$

$\mathrm{U}=64.9$ to $67.4 \%$

Dana VI, p. 978 


\section{B. MINERALS WITH MINOR AMOUNTS OF URANIUM AND THORIUM}

\section{ALLANITE}

$(\mathrm{Ca}, \mathrm{Ce}, \mathrm{Th})_{2}(\mathrm{Al}, \mathrm{Fe}, \mathrm{Mg})_{3} \mathrm{Si}_{3} \mathrm{O}_{12}(\mathrm{OH})$

$\mathrm{U}=0.017 \%, \mathrm{Th}=$ up to $3.2 \%$

Dana VI, pp. 522-526

Nagatelite - phosphatian variety of allanite

$\mathrm{Th}=0.8 \%$

Am. Mineralogist, vol. 16, pp. 343-344, 1931

Orthite - synonym of allanite

Ytro-orthife (also, yttrium orthite) - synonym of allanite.

Mineralog. Mag., vol. 23, p. 639, 1934

\section{ANTHRAXOLITE}

A hydrocarbon, nickeliferous and uraniferous; compare kolm and thucolite.

$\mathrm{U}=0.0029 \%$

Am. Mineralogist, vol. 19, pp. 426-428, 1934

Asphaltite, broggite, and cerburan are similar materials

\section{BASTNAESITE}

$(\mathrm{Ce}, \mathrm{La}) \mathrm{FCO}_{3}$

$\mathrm{U}$ and $\mathrm{Th}$ present but less than $1 \%$.

U. S. Geol. Survey, manuscript report; Dana VI, p. 291

\section{CAPPELENITE}

$(\mathrm{Ba}, \mathrm{Y})_{2} \mathrm{SiBO}_{6}$

$\mathrm{Th}=0.42 \%$

Dana VI, pp. 413-414

CERITE

$\mathrm{Ce}_{2} \mathrm{SiO}_{5}$

$\mathrm{U}=0.4 \%, \mathrm{Th}=0.3 \%$

Am. Mineralogist, vol. 25, pp. 381-404, 1940

\section{CHINGLUSUITE}

A complex silicate of $\mathrm{Na}, \mathrm{Mn}, \mathrm{Ca}$, and $\mathrm{Ti}$.

$\mathrm{Th}=0.05 \%$

Acad. sci. URSS Bull., pp. 153-157, 1938

\section{CORDYLITE}

$\mathrm{Ce}_{2} \mathrm{BaF}\left(\mathrm{CO}_{3}\right)_{3}$

$\mathrm{Th}=$ up to $0.27 \%$

Dana VI, appendix II, p. 31

CORVUSITE

$\mathrm{V}_{2} \mathrm{O}_{4} \cdot 6 \mathrm{~V}_{2} \mathrm{O}_{5} \cdot \mathrm{xH}_{2} \mathrm{O}$ ?

Validity of species is questionable.

$\mathrm{U}=1.0$ to $2.8 \%$

Am. Mineralogist, vol. 18, pp. 195-205, 1933

\section{DAVIDITE}

Contains $\mathrm{Ti}, \mathrm{Fe}$, rare earths, U, V, and $\mathrm{Cr}$.

$\mathrm{U}=8.1 \%, \mathrm{Th}=0.12 \%$

Dana VII, p. 542; Royal Soc. S. Australia

Trans., vol. 68, p. 334, 1944

\section{FERSMITE}

$(\mathrm{Ca}, \mathrm{Ce}, \mathrm{Na})(\mathrm{Cb}, \mathrm{Ti}, \mathrm{Fe}, \mathrm{Al})_{2}(\mathrm{O}, \mathrm{OH}, \mathrm{F})_{6}$

$\mathrm{Th}=\mathbf{0 . 4 2 \%}$

Acad. sci. URSS, C. R., 52, pp. 69-71, 1946

\section{HIELMITE}

$\mathrm{AB}_{2} \mathrm{O}_{6}$ or $\mathrm{A}_{2} \mathrm{~B}_{3} \mathrm{O}_{10}$

Also hjelmite.

$A=\mathrm{Y}, \mathrm{Fe}^{2}, \mathrm{U}^{4}, \mathrm{Mn}, \mathrm{Ca}$

$\mathrm{B}=\mathrm{Cb}, \mathrm{Ta}, \mathrm{Sn}, \mathrm{W}$

$\mathrm{U}=\mathbf{4 . 0}$ to $4.3 \%$

Dana VII, pp. 779-780

\section{HOKUTOLITE}

Variety of barite. Radioactive mixture of $\mathrm{Pb}$ and $\mathrm{Ba}$ sulfate. Probably contains $\mathrm{Ra}, \mathrm{Th}$, and $\mathrm{U}$.

Mineralog. Mag., vol. 16, p. 362, 1913

Anglesoborite - synonym of hokutolite JOHNSTRUPITE

A complex silicate of $\mathrm{Na}, \mathrm{Ca}, \mathrm{Th}, \mathrm{Ce}$, and $\mathrm{Ti}$.

$\mathrm{Th}=0.7 \%$

Dana VI, pp. 720-721 
KOLM

Rock with hydrocarbon and uranium. Form

in which uranium is present is unknown.

$\mathrm{U}=0.44 \%$

Am. Chem. Soc. Jour., vol. 52, pp. 4848-4851, 1930

See also anthraxolite and thucolite.

\section{LOVOZERITE}

Complex silicate of $\mathrm{Ti}$ and $\mathrm{Zr}$.

$\mathrm{Th}=0.50 \%$

Acad. sci. URSS, C. R. 25, p. 735, 1939

\section{MELANOCERITE}

Chiefly a boro-silicate of the $\mathrm{Ce}$ and $\mathrm{Y}$ metals.

$\mathrm{Th}=1.5 \%$

Dana VI, pp. 414-415

Caryocerife - near melanocerite

$\mathrm{Th}=12.0 \%$

Dana VI, p. 415

MICROLITE

$(\mathrm{Na}, \mathrm{Ca})_{2}(\mathrm{Ta}, \mathrm{Cb})_{2} \mathrm{O}_{6}(\mathrm{O}, \mathrm{OH}, \mathrm{F})$

In pyrochlore-microlite series.

$\mathrm{U}=$ up to $5.1 \%, \mathrm{Th}=0.2 \%$

Dana VII, pp. 748-754

Calciotantalite - possibly a mixture of microlite and tantalite.

Dana VII, p. 787

Haddamite - synonym of microlite

Dana VII, p. 748

Metasimpsonite - an alteration product of simpsonite, later identified with microlite.

Dana VII, p. 755

Neotantalite - an altered microlite with composition close to tantalite.

$\mathrm{U}=\mathrm{tr}$.

Dana VII, pp. 748, 750-751, 753

\section{MOSANDRITE}

Complex silicate of $\mathrm{Na}, \mathrm{Ca}, \mathrm{Ce}$, and $\mathrm{Ti}$.

$\mathrm{Th}={ }^{\prime} 0.3 \%$

Dana VI, pp. 721-722; Also, Zentralbl. Mineralogie, 1934, Abt. A, pp. 76-79,

\section{PISEKITE}

Essentially a columbate-tantalate-titanate of $U$ and rare earths, with Th and Sn. No quantitative analysis available. May be related to ampangabeite.

Dana VII, pp. 807-808

\section{PYROCHLORE}

$(\mathrm{Na}, \mathrm{Ca})_{2}(\mathrm{Cb}, \mathrm{Ta})_{2} \mathrm{O}_{6} \mathrm{~F}$

In pyrochlore-microlite series.

$\mathrm{U}=$ up to $14.0 \%$

$\mathrm{Th}=$ up to $4.4 \%$

Dana VII, pp. 748-754

Azorite - synonym of pyrochlore

Chalcolamprite - synonym of pyrochlore

Dana VII, pp. 748, 750, 754

Ellsworthite = composition is close to pyrochlore, but analyses show it is relatively high in $\mathrm{U}$ and $\mathrm{H}_{2} \mathrm{O}$ and low in alkalies.

$\mathrm{U}=17.1 \%$

Dana VII, pp. 748, 750-752

Endeiolite - similar in composition to chalcolamprite. An altered pyrochlore?

Dana VII, pp. 748, 754

Hatchettolite - uranian pyrochlore

$\mathrm{U}=14.0 \%, \mathrm{Th}=0.5 \%$

Dana VII, pp. 748, 750-752

Koppite - synonym of pyrochlore

Dana VII, pp. 748, 750, 752

Marignacite - synonym of pyrochlore

$\mathrm{Th}=0.2 \%$

Dana VII, pp. 748, 750-752

Pyrrhite - synonym of pyrochlore

Dana VII, pp. 748, 752, 754

\section{RINKITE}

Complex silicate of $\mathrm{Na}, \mathrm{Ca}, \mathrm{Ce}$, and $\mathrm{Ti}$.

$\mathrm{Th}=$ small amounts

Dana VI, p. 722

Kondrikite - a mixture of rinkite and zeolite; synonym of kondrikovite.

Lovchorrife - alteration product of rinkite?

$\mathrm{U}=$ up to $0.01 \%$

$\mathrm{Th}=$ up to $0.7 \%$ 
Yudyavrite - alteration product of rinkite.

$\mathrm{Th}=$ about $1 \%$

\section{RINKOLITE}

Complex silicate of $\mathrm{Na}, \mathrm{Ca}, \mathrm{Ce}$, and $\mathrm{Zr}$.

$\mathrm{Th}=$ up to $0.41 \%$

Am. Mineralogist, vol. 11, p. 289, 1926

Acad. s. URSS Bull. 20, p. 1181, 1926

\section{ROWLANDITE}

An yttrium silicate.

$\mathrm{U}=0.4 \%$

Dana VI, p. 1047

\section{STEENSTRUPINE}

Complex silicate of rare earths, $\mathrm{Th}, \mathrm{Na}, \mathrm{K}, \mathrm{Fe}$, $\mathrm{Mn}, \mathrm{Mg}, \mathrm{P}, \mathrm{Be}, \mathrm{Al}$, and $\mathrm{Ta}$, with $(\mathrm{OH})$ and $\mathrm{F}$.

$\mathrm{Th}=6.2 \%$

Dana VI, p. 415, Also, Neues Jahrb., BeilageBand 64, Abt. A, pp. 235-249, 1931

\section{TENGERITE}

$\mathrm{CaY}_{3}\left(\mathrm{CO}_{3}\right)_{4}(\mathrm{OH})_{3} \cdot 3 \mathrm{H}_{2} \mathrm{O}$ ?

Alteration product of yttrialite.

$\mathrm{Th}=0.3 \%$

Sci. Papers Inst. Phys. Chem. Res. (Tokyo), vol. 34, pp. 832-841, 1938

The originally described tengerife, Dana VI, pp. 306-307, is a different mineral, supposedly beryllium yttrium carbonate; no published analysis.

\section{THALENITE}

$\mathrm{Y}_{4} \mathrm{Si}_{4} \mathrm{O}_{13}(\mathrm{OH})_{2}$

Related to yttrialite.

$\mathrm{Th}=0.16 \%$

Dana VI, appendix I, p. 68

\section{THUCOLITE}

A hydrocarbon.

See kolm and anthraxolite.

$\mathrm{U}=4.9 \%, \mathrm{Th}=42.5 \%$

These percentages are from the ash which is only about 1 percent by weight of total material.

Am. Mineralogist, vol. 13, pp. 419-448, 1928

\section{TRITOMITE}

A boro-silicate of the cerium and yttrium metals, calcium, and thorium, also containing fluorine. Exact formula uncertain.

$\mathrm{Th}=7.5$ to $8.3 \%$

Dana VI, p. 416

\section{TSCHEFFKINITE}

Complex silicate of rare earths, $\mathrm{Fe}, \mathrm{Mn}, \mathrm{Mg}$, $\mathrm{Ca}, \mathrm{Al}, \mathrm{Ti}, \mathrm{Th}$, and $\mathrm{U}$.

Also chevkinite

$\mathrm{U}=2.3 \%, \mathrm{Th}=$ up to $18.4 \%$

Am. Mineralogist, vol. 31, pp. 582-588, 1946

\section{TURANITE}

A hydrated copper vanadate reported to contain $3.2 \% \mathrm{U}$; validity of species is questionable.

Acad. sci. St. Petersbourg, Bull. 3, p. 185, 1909

\section{VANOXITE}

A hydrated vanadium oxide, perhaps $2 \mathrm{~V}_{2} \mathrm{O}_{4} \cdot \mathrm{V}_{2} \mathrm{O}_{5} \cdot 8 \mathrm{H}_{2} \mathrm{O}$, reported to contain up to $0.5 \% \mathrm{U}$.

U. S. Geol. Survey Bull. 750, p. 63, 1924

\section{VOLBORTHITE}

$\mathrm{Cu}_{2}\left(\mathrm{VO}_{4}\right)(\mathrm{OH})$

$\mathrm{U}=3.1 \%$

Nenadkevich and Volkov, Acad. sci. URSS, C.R. 43, 1926

\section{Calciovolborthite}

$\mathrm{Cu}, \mathrm{Ca}\left(\mathrm{VO}_{4}\right) \mathrm{OH}$

Nenadkevich and Volkov, Acad. sci. URSS, C. R. 43,1926

\section{WIIKITE}

Ill-defined mixture and alteration product of minerals high in $\mathrm{Cb}, \mathrm{Ta}, \mathrm{Ti}, \mathrm{Si}$, and $\mathrm{Y}$.

Dana VII, p. 801

\section{Nuolaite}

A mixture similar to wiikite.

$\mathrm{Th}=1.8$ to $3.5 \%$

Dana VII, p. 801 
XENOTIME

$\mathrm{YPO}_{4}$

$\mathrm{U}=$ up to $3.6 \%$

$\mathrm{Th}=0.4$ to $2.9 \%$

Dana VI, pp. 748-749

\section{YTTRIALITE}

Silicate of $\mathrm{Th}$ and $\mathrm{Y}$ metals chiefly.

$\mathrm{U}=0.8 \%, \mathrm{Th}=10.5 \%$

Dana VI, p. 512

\section{YTTROCRASITE}

$(\mathrm{Y}, \mathrm{Th}, \mathrm{U}, \mathrm{Ca})_{2}(\mathrm{Ti})_{4} \mathrm{O}_{11}$ ?

$\mathrm{U}=2.3 \%, \mathrm{Th}=7.7 \%$

Dana VII, p. 793

\section{YTTROTANTALITE}

$(\mathrm{Fe}, \mathrm{Y}, \mathrm{U})(\mathrm{Cb}, \mathrm{Ta}) \mathrm{O}_{4}$

$\mathrm{U}=3.4$ to $3.9 \%$

$\mathrm{Th}=0.6$ to $0.7 \%$

Dana VI, pp. 763-764

Yttrocolumbite is similar

Am. Mineralogist, vol. 25, p. 155, 1940

\section{ZIRCON}

$\mathrm{ZrSiO}_{4}$

$\mathrm{U}$ and $\mathrm{Th}$ very low in most samples, but up to $2.7 \% \mathrm{U}$, and up to $13.1 \%$ Th reported.

Dana VI, pp. 482-486

\section{Alvite}

Variety of zircon, near cyrtolite.

$\mathrm{Th}=13.1 \%$ ?

Dana VI, pp. 487-488

\section{Calyptolite}

Probably altered zircon.

Dana VI, pp. 482, 486

\section{Cyrtolite}

Altered zircon, containing $\mathrm{U}, \mathrm{Th}, \mathrm{Y}$, and other rare earths.

$\mathrm{U}=$ up to $1.4 \%$

Dana VI, p. 487

Hagatalite - synonym of zircon

$\mathrm{U}=\mathrm{tr} ., \mathrm{Th}=1.3 \%$

Am. Mineralogist, vol. 11, p. 137, 1926

\section{Hoegtreitite}

May be alvite.

Am. Mineralogist, vol. 12, p. 97, 1927

Noegite - variety of zircon

$\mathrm{U}=2.4$ to $2.7 \%$

$\mathrm{Th}=2.5$ to $4.4 \%$

Chem. Soc. Japan Jour., vol. 42, p. 1, 1921 Also, Mineralog. Abs., vol. 2, p. 36, 1923

\section{Oerstedite}

Altered zircon.

Dana VI, p. 486

Oyamalite - variety of zircon

$\mathrm{Th}=0.5 \%$

Am. Mineralogist, vol. 11, pp. 137-138, 1926

\section{Tachyaphaltite}

Probably altered zircon.

Dana VI, p. 486

Yamagutilite - variety of zircon

Also, yamaguchilite

Contains $\mathrm{P}_{2} \mathrm{O}_{5}$ and rare earths.

Mineralog. Mag., vol. 24, p. 626, 1937

\section{ZIRKELITE}

$(\mathrm{Ca}, \mathrm{Fe}, \mathrm{Th}, \mathrm{U})_{2}(\mathrm{Ti}, \mathrm{Zr})_{2} \mathrm{O}_{5}$ ?

$\mathrm{U}=1.4 \%, \mathrm{Th}=6.4 \%$

Dana VII, p. 740 


\section{MINERALS THAT SHOULD BE TESTED FOR URANIUM AND THORIUM}

\section{ABUKUMALITE}

CaY phosphate silicate

Am. Mineralogist, vol. 24, p. 66, 1939

\section{AMBATOARINITE}

Carbonate of $\mathrm{Sr}$ and rare earths.

Mineralog. Abs., vol. 1, p. 148, 1920

\section{ANCYLITE}

$\mathrm{Sr}_{3} \mathrm{Ce}_{4}\left(\mathrm{CO}_{3}\right)_{7}(\mathrm{OH}) .3 \mathrm{H}_{2} \mathrm{O}$ ?

Dana VI, appendix II, pp. 5-6

Weibyite

Related to ancylite

Dana VI, pp. 291-292

\section{BAZZITE}

Silicate of scandium, with $\mathrm{Fe}, \mathrm{Na}$, and rare earths.

Mineralog. Abs., vol. 8, p. 105, 1941

\section{BECKELITE}

$\mathrm{Ca}_{3}(\mathrm{Ce}, \mathrm{La}, \mathrm{Pr}, \mathrm{Nd})_{4} \mathrm{Si}_{3} \mathrm{O}_{15}$

Dana VI, appendix II, p. 14

\section{BRITHOLITE}

A cerium-silicate apatite?

Dana VI, appendix II, pp. 19-20

\section{BUSZITE}

Silicate of rare earths

Am. Mineralogist, vol. 14, pp. 438-439, 1929

\section{CENOSITE}

$2 \mathrm{CaO} .(\mathrm{Ce}, \mathrm{Y})_{2} \mathrm{O}_{3} \cdot \mathrm{CO}_{2} \cdot 4 \mathrm{SiO}_{2} \cdot \mathrm{H}_{2} \mathrm{O}$ ?

Also kainosite

Am. Mineralogist, vol. 15, pp. 205-219, 1930

\section{CHURCHITE}

A hydrous phosphate of cerium and calcium

Dana VI, p. 820

\section{ERDMANNITE}

Hydrous silicate of $\mathrm{Ce}, \mathrm{Y}, \mathrm{Fe}, \mathrm{Mn}, \mathrm{Al}$, and $\mathrm{Ca}$.
A mixture of homilite with a mineral in the melanocerite group?

Dana VI, pp. 416, 507

\section{ERIKITE}

Phosphate-silicate of rare earths, $\mathrm{Al}, \mathrm{Ca}$, and $\mathrm{Na}$.

Mineralog. Mag., vol. 14, p. 348, 1907

\section{EUDIALYTE}

$\mathrm{X}_{5} \mathrm{Y}_{2} \mathrm{Si}_{8} \mathrm{O}_{18}(\mathrm{Oh}, \mathrm{Cl})$

$\mathrm{X}=\mathrm{Ca}, \mathrm{Na}, \mathrm{Ce}$, etc.

$\mathrm{Y}=\mathrm{Z} \mathbf{r}, \mathrm{Fe}, \mathrm{Cb}$, etc.

Dana VI, pp. 409-412

Eucolite

Same as eudialyte, but with different optical sign.

Dana VI, pp. 409-412

\section{FLORENCITE}

$\mathrm{CeAl}_{3}\left(\mathrm{PO}_{4}\right)_{2}(\mathrm{OH})_{6}$

Dana VI, appendix II, p. 42

\section{FLUOCERITE}

$(\mathrm{Ce}, \mathrm{La}) \mathrm{F}_{3}$

Am. Mineralogist, vol. 6, p. 119, 1921

Tysonite $=$ fluocerite

Dana VI, p. 166

\section{GADOLINITE}

$\mathrm{Be}_{2} \mathrm{FeY}_{4} \mathrm{Si}_{2} \mathrm{O}_{13}$

Dana VI, pp. 509-512

\section{HELLANDITE}

$\mathrm{Ca}_{2}(\mathrm{Ce}, \mathrm{Al}, \mathrm{Mn})_{8} \mathrm{Si}_{4} \mathrm{O}_{1}{ }_{8} 3 \mathrm{H}_{2} \mathrm{O}$

Mineralog. Mag., vol. 13, p. 368, 1903

\section{LANTHANITE}

$\mathrm{La}_{2}\left(\mathrm{CO}_{3}\right)_{2} \cdot 9 \mathrm{H}_{2} \mathrm{O}$

Dana VI, pp. 302-303,

\section{LESSINGITE}

Silicate of the rare earths.

Mineralog. Abs., vol. 10, p. 245, 1948 


\section{LORANSKITE}

$(\mathrm{Y}, \mathrm{Ce}, \mathrm{Ca})(\mathrm{Ta}, \mathrm{Zr}) \mathrm{O}_{4}$ ?

Dana VII, p. 767

\section{NUEVITE}

Titano-columbate of $\mathrm{Y}, \mathrm{Fe}$, and Ta. Validity of species is questionable.

Am. Mineralogist, vol. 32, pp. 204-205, 1947

\section{PARISITE}

$\mathrm{CaCe}_{2} \mathrm{~F}_{2}\left(\mathrm{CO}_{3}\right)_{3}$ ?

Dana VI, pp. 290-291

Synchisite

$\mathrm{CaCeF}\left(\mathrm{CO}_{3}\right)_{2}$

Related to parisite.

Mineralog. Mag., vol. 13, pp. 207-208, 1902

\section{PEROVSKITE GROUP}

\section{Perovskite}

$\mathrm{CaTiO}_{3}$ - some analyses show rare earths.

Dana VII, pp. 730-734

Dysanalyte - synonym of perovskite; analygis shows rare earths.

Dana VII, pp. 730-732

Knopite - in perovskite group; analysis shows rare earths.

Dana VII, pp. 730-733

Loparite - end member in perovskite-knopiteloparite series.

Perhaps $(\mathrm{Na}, \mathrm{Ce}, \mathrm{Ca})_{2}(\mathrm{Ti}, \mathrm{Cb})_{2} \mathrm{O}_{8}$

Dana VII, Pp. 730, 732-734

\section{RETZIAN}

Basic arsenate of $\mathrm{Mn}, \mathrm{Cu}$, and rare earths.

Dana VI, appendix I, p. 59

\section{RHABDOPHANITE}

Hydrous phosphate of $\mathrm{Ce}$ and $\mathrm{Y}$ groups; perhaps $(\mathrm{Ce}, \mathrm{Y}) \mathrm{PO}_{4} \cdot \mathrm{H}_{2} \mathrm{O}$

Also rhabdophane

Dana VI, p. 820

Scovillite - synonym of rhabdophanite

Dana VI, p. 820

\section{SCHETELIGITE}

$(\mathrm{Ca}, \mathrm{Y}, \mathrm{Sb}, \mathrm{Mn})_{2}(\mathrm{Ti}, \mathrm{Ta}, \mathrm{Cb})_{2}(\mathrm{O}, \mathrm{OH})_{7}$

Dana VII, p. 757

\section{SPHENE}

Essentially $\mathrm{CaTiSi}_{2} \mathrm{O}_{5}$, but commonly contains rare earths, columbium, and other elements.

Am. Mineralogist, vol, 32, pp. 637-642, 1947

Keilhavite $=$ yttrian sphene

Dana VI, p. 717

Titanite - synonym of sphene

Ytrotitanite - synonym of keilhauite

\section{THORTVEITITE}

$(\mathrm{Sc}, \mathrm{Y})_{2} \mathrm{Si}_{2} \mathrm{O}_{7}$

Am. Mineralogist, vol. 7, pp. 195-196, 1922

Befanamite - synonym of thortveitite

Am. Mineralogist, vol. 11, p. 137, 1926

TÓRNEBOHNTE

Chiefly $\mathrm{R}_{3}(\mathrm{~F}, \mathrm{OH})\left(\mathrm{SiO}_{4}\right)_{2}$

$\mathrm{R}=\mathrm{Ce},(\mathrm{La}, \mathrm{ND}), \mathrm{Al}, \mathrm{Fe}, \mathrm{Mn}, \mathrm{Mg}, \mathrm{Ca}$

Am. Mineralogist, vol. 6, pp. 118-119, I921

\section{WEINSCHENKITE}

$\mathrm{YPO}_{4} \cdot 2 \mathrm{H}_{2} \mathrm{O}$

Am. Mineralogist, vol. 29, pp. 97-107, 1944 
D. MINERALS THAT ARE NON-URANIUM- OR NON-THORIUM-BEARING, BUT THAT HAVE BEEN REPORTED TO CONTAIN IMPURITIES OR INTERGROWTHS OF URANIUM, THORIUM, OR RARE-EARTH MINERALS

\section{BADDELEYITE}

$\mathrm{ZrO}_{2}$

Analyses show traces of rare earths.

Dana VII, pp. 607-610

Brazilite - mixture of fibrous baddeleyite, zircon, altered zircon, and other minerals

Dana VII, p. 610

Caldasite - mixture of baddeleyite, zircon, altered zircon, and other minerals.

Dana VII, p. 610

Zirkite - mixture of baddeleyite, zircon, altered zircon and other minerals.

\section{COLUMBITE-TANTALITE SERIES}

\section{Columbite}

$(\mathrm{Fe}, \mathrm{Mn})(\mathrm{Cb}, \mathrm{Ta})_{2} \mathrm{O}_{6}$

$\mathrm{U}=$ up to $1.7 \%$

Dana VII, pp. 780-785

Baierite - synonym of columbite

Also baierine

Dana VII, p. 780

Dianite - synonym of columbite

Dana VII, p. 780

Ferrocolumbite - synonym of columbite

Dana VII, pp. 780, 783

Ferro-ilmenite - synonym of columbite

Dana VIl, pp. 780, 785

Ferrotantalite - synonym of tantalite

Dana VII, pp. 780, 783

Greenlandite - synonym of columbite

Dana VII, pp. 780, 784

Hermannolite - synonym of columbite

Dana VII, pp. 780, 785

Ildefonsite - synonym of tantalite

Dana VII. p. 780

Manganocolumbite - variety of columbite

Dana VII, pp. 780, 783-784
Manganotantalite - synonym of tantalite

Dana VII, pp. 780, 783-784

Siderotantalife - synonym of tantalite

Dana VII, p. 780

Tantalite

$(\mathrm{Fe}, \mathrm{Mn})(\mathrm{Ta}, \mathrm{Cb})_{2} \mathrm{O}_{6}$

\section{FLUORITE}

$\mathrm{CaF}_{2}$

Some samples, especially those of deeppurple color show radioactivity. This generally can be shown to be due to inclusions of uranium minerals, but the fluorite itself may possibly contain traces of uranium.

Dana VI, pp. 161-164

\section{Ytrocerite}

$\mathrm{CaF}_{2}$ with varying amounts of $(\mathrm{Y}, \mathrm{Ce}) \mathrm{F}_{3}$.

Dana VI, p. 182

Ythofluorite - yttrian variety of fluorite

Mineralog. Mag., vol. 16, p. 376, 1913

\section{FRANCOLITE}

An apatite which is an important constituent of many phosphate rocks, but which analyses show contains very little rare earths.

Dana VI, pp. 762, 764

\section{ILMENORUTILE}

Columbian rutile.

Dana VII, pp. 554, 557, 560

\section{KALKOWSKITE}

$\mathrm{Fe}_{2} \mathrm{Ti}_{3} \mathrm{O}_{8}$ ?

Analysis shows rare earths.

Dana VII, pp. 773-774

\section{KATANGITE}

$\mathrm{H}_{2} \mathrm{CuSiO}_{4}+\mathrm{H}_{2} \mathrm{O}$ ?

Is identical with chrysocolla; found together with uranium minerals in Katanga, Belgian Congo.

Am. Mineralogist, vol. 8, p. 39, 1923 
OPAL

$\mathrm{SiO}_{2} \cdot \mathrm{nH}_{2} \mathrm{O}$

Some varieties show green fluorescence due to uranium content.

\section{PYROMORPHITE}

$\mathrm{Pb}_{5}\left(\mathrm{PO}_{4}\right)_{3} \mathrm{Cl}$

Some specimens are uraniferous.

Zeitschr. Kristallographie, vol. 62, pp. 177-178, 1925

\section{SCAPOLITE}

$(\mathrm{Na}, \mathrm{Ca})_{4} \mathrm{Al}_{3}(\mathrm{Al}, \mathrm{Si})_{3} \mathrm{Si}_{6} \mathrm{O}_{24}\left(\mathrm{Cl}, \mathrm{CO}_{3}, \mathrm{SO}_{4}\right)$

A fluorescent variety contains $0.023 \% \mathrm{U}$.

Chemie der Erde, vol. 9, pp. 139-144, 1934-35

\section{SEFSTRÖMITE}

Mixture largely of ilmenite, similar to davidite.

Dana VII, p. 542
STRUEVERITE

Tantalian rutile.

Dana VII, pp. 554, 557-560

\section{TAPIOLITE SERIES}

Cassiterofantalite - synonym of ixiolite

Dana VII, p. 778

Ixiolite - may be equal to tapiolite

Dana VII, p. 778

Ixionolite - synonym of ixiolite

Mossite

$\mathrm{Fe}(\mathrm{Cb}, \mathrm{Ta})_{2} \mathrm{O}_{6}$

Dana VII, pp. 775-777

Skogbölife - synonym of tapiolite

Dana VII, pp. 775-778

Tapiolite

$\mathrm{FeTa}_{2} \mathrm{O}_{6}$

Dana VII, pp. 775-777 


\section{INDEX}

(A) indicates minerals containing uranium or thorium as major constituents.

(B) indicates minerals containing minor amounts of uranium or thorium.

(C) indicates minerals that should be tested for uranium and thorium content.

(D) indicates minerals that are non-uranium- or non-thorium-bearing, but that have been reported to contain impurities or intergrowths of uranium, thorium, or rare-earth minerals.

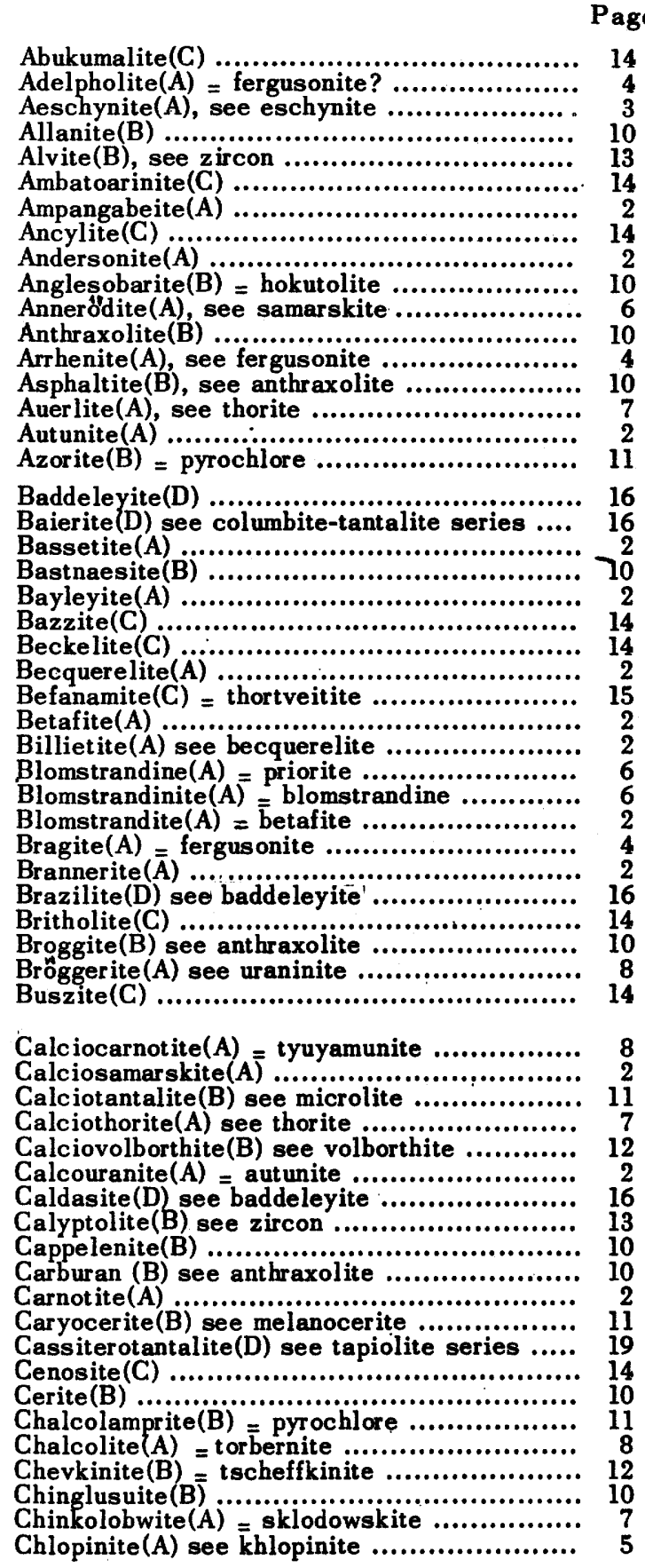

Chlorothorite $(A)=$ thorogummite $\ldots . . \ldots \ldots \ldots \ldots . .8$

Churchite(C) ..................................... 14

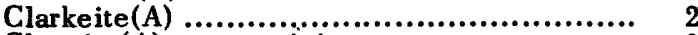

Cleveite (A) see uraninite .......................... 8

Columbite(D) see columbite-tantalite series .. 16

Columbite-tantalite series (D) ................. 16

Coracite(A) $=$ uraninite $\ldots \ldots \ldots \ldots \ldots \ldots \ldots \ldots \ldots . .6 \%$

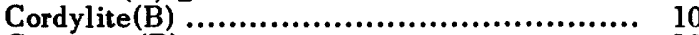

Corvusite(B) ................................ 10

Cryptolite $(A)=$ monazite ........................ 5

Cuprosklodowskite(A) ........................... 2

Cupro-uranite $(A)=$ torbernite .................... 8

Curite (A) .......................................... 3

Cyrtolite(B) see zircon .......................... 13

Dakeite $(A)=$ schroeckingerite $. . . \ldots . . . \ldots \ldots \ldots \ldots . . .7$

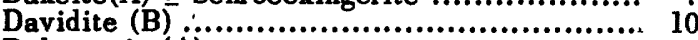

Delorenzite $(A)$........................................... 3

Dewindtite(A) .......................................... 3

Dianite(D) see columbite-tantalite series ...... 16

Diderichite(A) ........................................ 3

Djalmite (A) .............................................. 3

Droogmansite(A) .................................. 3

Dumontite(A) ..................................... 3

Dysanalyte $(C)=$ perovskite ......................... 15

Edwardsite $(A)=$ monazite $. . . . \ldots \ldots \ldots \ldots \ldots \ldots . .5 \quad 5$

Eliasite $(A)=$ gummite .............................. 4

Ellsworthite $\overline{\bar{B}}$ B) see pyrochlore .................. 11

Enalite(A) see thorite .............................. 7

Endeiolite(B)' see pyrochlore ....................... 11

Epiianthinite(A) ................................... 3

Erdmannite(C) ......................................... 14

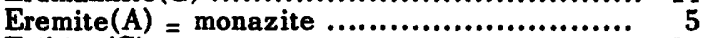

Erikite $(\mathrm{C})$........................................ 14

Eschwegite $(A)$ see euxenite .......................... 3

Eschynite(A) ...................................... 3

Eucolite(C) see eudialyte ............................. 14

Eucrasite $(A)$ see thorite ............................ 7

Eudialyte(C) …........................................ 14

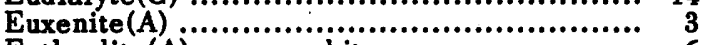

Eytlandite $(A)=$ samarskite $\ldots . . . \ldots \ldots \ldots \ldots \ldots \ldots \ldots . .66$

Ferghanite(A) see tyuyamunite .................. 8

Fergusonite $(A)$................................... 4

Ferrocolumbite(D) see columbite-tantalite series ........................................... 16

Ferro-ilmenite(D) see columbite-tantalite series ......................................... 16

Ferrotantalite(D) see columbite-tantalite series ............................................ 16

Ferrothorite $(\mathrm{A})$ see thorite ............................ 7

Fersmite(B) ........................................... 10

Florencite(C) ......................................... 14

Fluocerite(C) ................................... 14

Fluorite(D) ........................................ 16

Flutherite $(A)=$ liebigite ......................... 5

Formanite(A) ......................................... 4

Fourmarierite $(A)$. .................................... 4

Francolite(D) ................................... 16

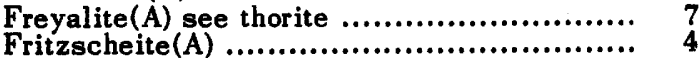


Gilpinite(A) $=$ johannite ......................... 5

Greenlandite $(\bar{D})$ see columbite-tantalite

series

Gummite (A)

Haddamite $(B)=$ microlite

Hagatalite $(B)=$ zircon ..........................

Hatchettolite $(\overline{\bar{B}})$ see pyrochlore ....................

Hellandite $(C)$

Hermannolite(D) see columbite-tantalite series

Hielmite $(\ddot{B})$

Hlopinite(A) see khlopinite ............................

Hoegtveitite(B) see zircon ........................

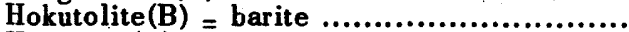

Huttonite $(A)$

Hyblite(A) see thorite ............................

Hydro-euxenite $(A)=$ ampangabeite ................

Hydrosamarskite $(A)$ see samarskite .............

Hydrothorite(A) see mackintoshite ...............

Ianthinite(A)

Ildefonsite(D) see columbite-tantalite series

Ilmenorutile(D)

Ixiolite(D) see tapiolite series ......................

Ixionolite(D) see tapiolite series

Jachymovite $(A)=$ cuprosklodowskite ............

Johannite(A) ...........................................

Johnstrupite(B) ....

Kainosite $(C)=$ cenosite

Kalkowskite(D)

Kasolite(A)

Katangite(D)

Keilhauite(C) see sphene.....

Khlopinite(A) ........................................

Knopite(C) see perovskite group ....................

Kochelite $(A)=$ fergusonite $\ldots . . . \ldots \ldots \ldots \ldots \ldots . . . . .$.

$\mathrm{Kolm}(\mathrm{B})$

Kondrikovite $(B)=$ kondrikite see rinkite .......

Koppite $(B)=$ pyrochlore $\ldots . . . . . . . . . . . . . . . . . . . . . .$.

Lambertite $(A)=$ uranophane

Lanthanite(C)

Lessingite(C) .......................................

Loparite(C) see perovskite group ...................

Loranskite(C) .

Lovchorrite(B) see rinkite .........................

Lovozerite(B)

Lyndochite(A) see euxenite .......................

Mackintoshite(A)

Maitlandite (A)

Manganocolumbite(D) see columbitetantalite series

Manganotantalite(D) see columbite-

tantalite series

Marignacite $(B)=$ pyrochlore ..........................

Masuyite (A)

Med jidite (A) ....................................

Melanocerite $(B)$

Mendeleyevite $(A)$ see betafite ...................

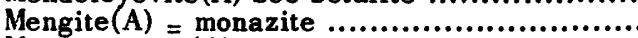

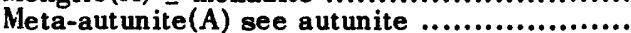

Metachalcolite(A) see torbernite .................

Metasimpsonite(B) see microlite .................

Metatorbernite $(A)$ see torbernite .................

Microlite(B)

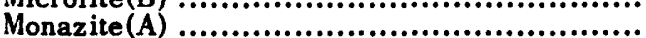

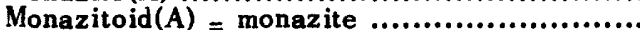

Mosandrite(B) ...................................

Mossite(D) see tapiolite series
Naegite $(B)=$ zircon $\ldots \ldots \ldots \ldots \ldots \ldots \ldots \ldots \ldots \ldots . . .13$

Nagatelite $(\bar{B})$ see allanite ...................... 10

Nasturan $(A)$ see uraninite .......................... 8

Neotantalite(B) see microlite ................. 11

Nicolayite $(A)$ see maitland ite .................... 5

Nivenite(A) see uraninite .......................... 8

Nohlite(A) ........................................ 6

Nuevite $(C)$............................................ 15

Nuolaite(B) see wiikite .......................... 12

Oerstedite(B) see zircon ....................... 13

Opal(D) ...................................... 17

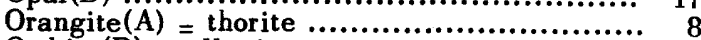

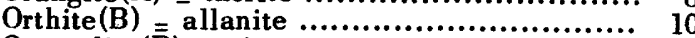

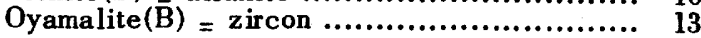

Paraschoepite (A) ................................ 6

Parisite(C) ..................................... 15

Parsonsite (A),........................................ 6

Perovskite(C) see perovskite group ............. 15

Perovskite group(C) .............................. 15

Phosphocerite $(A)=$ monazite ...................... 5

Phosphuranylite(A) ................................. 6

Pilbarite(A) see mackintoshite ..................... 5

Pisekite(B) .................................... I1

Pitchblende(A) see uraninite ..................... 8

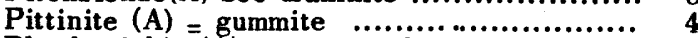

Plumboniobite $(\AA)$ see samarskite ................ 6

Polycrase(A) ........................................... 6

Polymignyte $(\mathrm{A})$....................................... 6

Priorite $(A)$............................................ 6

Pyrochlore(B) .................................. 11

Pyromorphite(D) $\ldots \ldots \ldots \ldots \ldots \ldots \ldots \ldots \ldots \ldots \ldots \ldots \ldots \ldots \ldots \ldots, \quad 17$

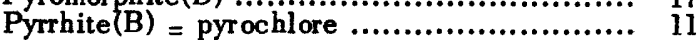

Randite(A) see uranophane ..................... 9

Rauvite(A) ...................................... 6

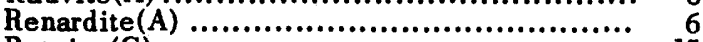

Retzian(C) ........................................... 15

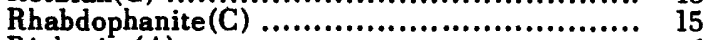

Richetite (A) ......................................... 6

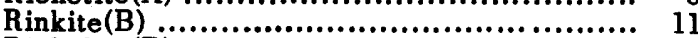

Rinkolite $(B) \ldots \ldots \ldots \ldots \ldots \ldots \ldots \ldots \ldots \ldots \ldots \ldots \ldots \ldots \ldots, 12$

Risörite $(A)=$ fergusonite ......................... 4

Rogersite $(A)=$ samarskite $\ldots \ldots \ldots \ldots \ldots \ldots \ldots \ldots \ldots \ldots . .6 \%$. 6

Rowlandite $(B)$ (..................................... 12

Rutherfordine(A) .................................. ${ }_{6}$

Rutherfordite(A) see fergusonite ............... 4

Saleeite(A) ......................................... 6

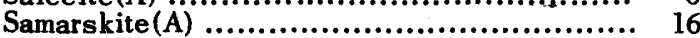

Samirésite(A) = betafite $\ldots . . \ldots \ldots \ldots \ldots \ldots \ldots \ldots \ldots . . . .19$

Scapolite(D) ........................................ 17

Scheteligite $(\dddot{C})$.................................... 15

Schoepite(A) ........................................ ${ }_{7}$

Schroeckingerite $(\mathrm{A})$............................... 7

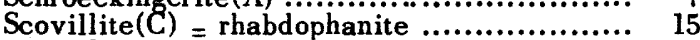

Sefströmite(D) $\ldots \ldots \ldots \ldots \ldots \ldots \ldots \ldots \ldots \ldots \ldots \ldots \ldots, \quad 17$

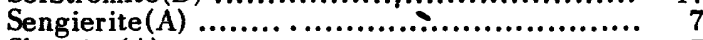

Sharpite(A) ........................................ 7

Siderotantalite(D) see columbite-tantalite

series ............................................ 16

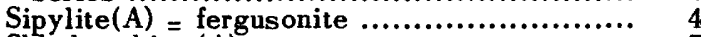

Sklodowskite $(A) \ldots \ldots \ldots \ldots \ldots \ldots \ldots \ldots \ldots \ldots \ldots \ldots \ldots, 7$

Skogbölite(D) see tapiolite series .............. 17

Soddyite(A) ........................................ $r_{7}$

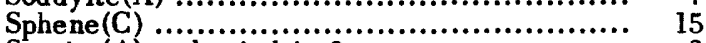

Stasite $(A)=$ dewindtite ? .......................... 3

Steenstrupine(B) ................................ 12

Strueverite(D) $\ldots \ldots \ldots \ldots \ldots \ldots \ldots \ldots \ldots \ldots \ldots \ldots \ldots \ldots, 17$

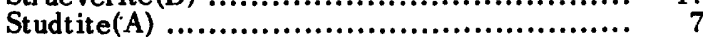

Swartzite(A) ....................................... 7

Synchisite(C) see parisite .................... 15

Tachyaphaltite(B) see zircon .................. 13

Tantalite(D) see columbite-tantalite

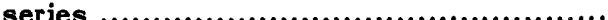

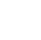

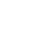


Tanteuxenite(A) see euxenite ................. 3

Tapiolite(D) see tapiolite series ............... 17

Tapiolite series(D) ........................... 17

Tengerite(B) .................................... 12

Thalenite(B) ............................... 12

Thorianite(A) .................................... 7

Thorite(A) ..................................... 7

Thorogummite(A) ........................... 8

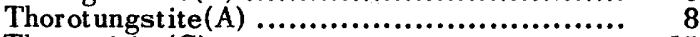

Thortveitite(C) .............................. 15

Thucolite(B) ...................................... 12

Titanite $(\mathrm{C})=$ sphene $\ldots \ldots \ldots \ldots \ldots \ldots \ldots \ldots \ldots . . .15$

Toddite(A) see euxenite ........................ 3

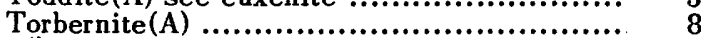

Törnebohmite(C) ............................. 15

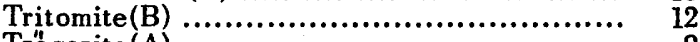

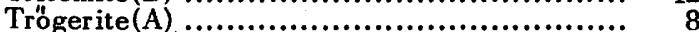

Tscheffkinite(B) .................................. 12

Turanite(B) .................................... 12

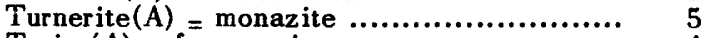

Tyrite $(A)=$ fergusonite .......................... 4

Tysonite $(\bar{C})=$ fluocerite $\ldots \ldots \ldots \ldots \ldots \ldots \ldots \ldots \ldots .14$

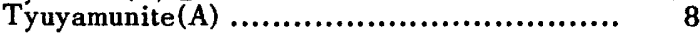

Ulrichite $(A)=$ uraninite $\ldots \ldots \ldots \ldots \ldots \ldots \ldots \ldots \ldots . .8$

Uraconite $(A)$.................................... 8

Uraninite $(A)$................................. 8

Uranite $(A)=$ torbernite- autunite group ........ 8

Uranochalcite(A) see cuprosklodowskite ..... 3

$\operatorname{Uranocher}(A)=$ uraconite....................

Uranocircite $(A \overline{)}$

Uranolepidite $(A)$ = vandenbrandeite ..........

Uranoniobite $(A)=$ uraninite

Uranophane (A)

Uranophyllite $(A)=$ torbernite $\ldots \ldots \ldots \ldots . . . . .$.

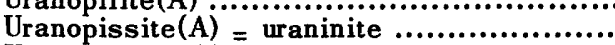

Uranospathite $(A)$

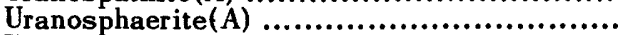

Uranospinite(A)

Uranotantalite $(A)=$ samarskite
Vandenbrandeite(A)

Vandendriesscheite $(\ddot{A})$

Vanoxite(B)

Vietinghofite $(\AA)=$ samarskite ................... 7

Voglianite(A) see cuprosklodowskite ......... 3

Voglite (A) ......................................... 9

Volborthite(B) .................................... 12

Vudyavrite(B) see rinkite ...................... 12

Walpurgite(A) ..................................... . 9

Weibyite(C) see ancylite ........................ 14

Weinschenkite(C) ................................ 15

Wiikite(B) ...................................... 12

Xenotime(B) ................................... 13

Yamaguchilite $(B)=$ yamagutilite $. . . \ldots \ldots \ldots \ldots . .13$

Yamagutilite $(B)=$ zircon $. . . \ldots \ldots \ldots \ldots \ldots \ldots \ldots . .13$

Yttrialite(B) ...................................... 13

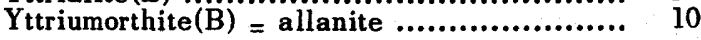

Uranothallite $(A)=$ liebigite $\ldots . \ldots \ldots \ldots \ldots \ldots \ldots . \quad 5$

Uranothorianite $(\overline{\bar{A}})$ see thorianite . ............ 7

Uranothorite(A) see thorite ...................... . 8

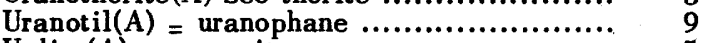

$\operatorname{Urdite}(A)=$ monazite $\ldots \ldots \ldots \ldots \ldots \ldots \ldots \ldots \ldots \ldots . .5$

Uvanite $(A)$.................................... 9

Yttrocerite(D) see fluorite ...................... 16

Yttrocolumbite(B) see yttrotantalite ........... 13

Yttrocrasite(B) .................................. 13

Yttrofluorite(D) see fluorite .................... 16

Yttrogummite $(A)=$ gummite $\ldots . . . . . . . . . . . . . . .4 \quad 4$

Yttro-ilmenite $(A)=$ samarskite $\ldots \ldots \ldots \ldots \ldots \ldots . .7$

Yttro-orthite $(B)=$ allanite $. . . . \ldots \ldots \ldots \ldots \ldots \ldots . . .10$

Yttrotantalite(B) .................................. 13

Yttrotitanite $(\mathrm{C})=$ keilhauite $. . . \ldots \ldots \ldots \ldots \ldots \ldots . \quad 15$

Zeunerite(A) ................................... 9

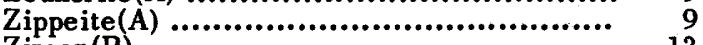

Zircon(B) ........................................ 13

Zirkelite(B) ....................................... 13

Zirkite(D) see baddeleyite ...................... 16 